\title{
Beam-dynamic effects of a droop in an induction accelerating voltage
}

\author{
Yoshito Shimosaki, Eiji Nakamura, and Ken Takayama \\ Accelerator Laboratory, High Energy Accelerator Research Organization (KEK), 1-1 Oho, Tsukuba, Ibaraki 305-0801, Japan \\ Kota Torikai \\ Faculty of Engineering, Kyushu University, 6-10-1 Hakozaki, Fukuoka-shi, Fukuoka 812-8581, Japan
}

\begin{abstract}
Masao Watanabe
Beam Physics and Engineering Laboratory, The Institute of Physical and Chemical Research (RIKEN), 2-1 Hirosawa, Wako, Saitama 351-0198, Japan
\end{abstract}

\author{
Mitsuo Nakajima and Kazuhiko Horioka \\ Department of Energy Science, Interdisciplinary Graduate School at Nagatsuta, Tokyo Institute of Technology, \\ Nagatsuta 4259, Yokohama 226-8502, Japan \\ (Received 26 August 2003; published 13 January 2004)
}

\begin{abstract}
Proof-of-principle experiments on the induction synchrotron concept are scheduled using the KEK $12-\mathrm{GeV}$ proton synchrotron, in which rf bunches and a superbunch will be accelerated with a long step voltage generated in the induction accelerating gaps. An unavoidable droop in the induction voltage gives an additional focusing or defocusing force in the longitudinal direction. It largely deforms the barrier bucket confining the superbunch, leading to a nonuniform particle distribution. The effects are serious in an induction synchrotron with a transition energy. Longitudinal emittance blowup beyond the transition energy is not acceptable. The necessity of compensating for the droop is discussed.
\end{abstract}

DOI: $10.1103 /$ PhysRevSTAB.7.014201

PACS numbers: 41.85.Ct, 29.20.-c

\section{INTRODUCTION}

Superbunch acceleration is a key concept in an induction synchrotron $[1,2]$. In an induction synchrotron, superbunches confined in the longitudinal direction by a pair of barrier voltages are accelerated with long induction step voltage pulses as schematically shown in Fig. 1.

Experiments for proof of principle (POP) of the induction synchrotron are planned using the KEK $12-\mathrm{GeV}$ proton synchrotron during 2003-2007 [3]. The experiments will proceed step by step. In the first step, a single rf bunch that is captured in the existing rf will be accelerated with the induction voltage alone. For this purpose, four newly developed induction accelerating cavities [4] with an output voltage of $2.5 \mathrm{kV} /$ each will be installed during the 2003 winter shutdown. As the second step, an induction barrier experiment is planned, where 1-9 booster rf bunches are injected into the main ring, immediately captured in the induction barrier bucket, and then merged into a single superbunch. Various beam-handling exercises, such as adiabatic moving of the superbunch, will be performed. In the last step, a superbunch will be accelerated up to the flattop energy with the induction voltage.

The induction acceleration device can be thought of as a series of one-to-one transformers driven by low-impedance pulse sources in which the beam acts as the secondary. In an equivalent-circuit model, the induction cavity is a parallel circuit of inductance, resistance, and capacitance, standing for the magnetic core, core loss and other losses, and acceleration gap, respectively. The cavity is connected to a high-voltage pulse modulator through a matching cable and driven at a repetition rate on the order of $\mathrm{MHz}$ [5]. Some droop in the output voltage, which is generated across the inductance, is generally unavoidable because of a finite inductance and resistance. Our preliminary measurement has indicated a droop of several to $10 \%$. It is easily supposed that the droop voltage gives accelerating particles an extra defocusing effect in the longitudinal direction below the transition energy and a focusing effect above the transition energy. It tends to deform the desired barrier bucket shape. The purpose of

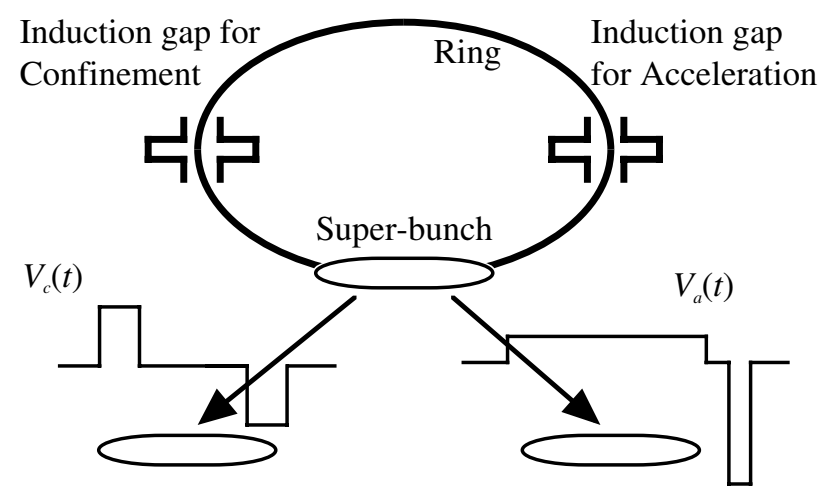

FIG. 1. Schematic view of the induction synchrotron. $V_{c}$ is the barrier voltage for the longitudinal confinement and $V_{a}$ is the accelerating voltage. 
this paper is to theoretically manifest its beam-dynamical effects and estimate its significance in the synchrotron oscillation of the superbunch. In addition, a discussion will develop concerning the case of induction acceleration of $\mathrm{rf}$ bunches, to which we will soon face.

The organization of this paper is as follows. In Sec. II, the droop voltage is evaluated as a function of the circuit parameters through an equivalent-circuit analysis of the induction accelerating device. In Sec. III, the theory of synchrotron oscillation with droop is developed. In Sec. IV, results of particle tracking through an entire acceleration are discussed. Significant emittance blowup at a transition is realized in the case of a superbunch. In Sec. V, a possible way to compensate for the droop is proposed. Last, newly realized knowledge is summarized.

\section{EQUIVALENT-CIRCUIT MODEL OF THE INDUCTION CAVITY AND DROOP VOLTAGE}

As is well understood, an induction accelerator is a pulsed passive device. In order to generate a step voltage in the accelerating gaps, the accelerating cavity is energized with a pulse modulator employing power metaloxide-semiconductor field-effect transistors as switching elements, which are connected to a dc power supply. Switching of the modulator has to be synchronized with the revolution of beams; it is operated at a high repetition rate on the order of $1 \mathrm{MHz}$. The generating peak voltage is somewhat less than the voltage of the bank capacitance of the modulator because of a finite resistance of the transmission cable. An equivalent circuit of the induction unit consisting of the condenser bank, power-transmission cable, and the induction cavity can be expressed as shown in Fig. 2, in which $Z_{t}, L, C$, and $R$ represent the characteristic impedance of a power-transmission cable with length $l$, the core inductance, the stray capacitance of the induction unit, and the total resistance of the core loss $R_{C}$ and the matching load $R_{M}$, respectively. To reduce the reflection, a matching load is connected parallel to the cavity. The quasimatching condition is expressed as $1 /(\omega L)+\omega C+1 / R_{M}+1 / R_{C}=1 / Z_{t}$, where $f=$ $2 \pi / \omega$ is the dominant frequency of the step voltage pulse.

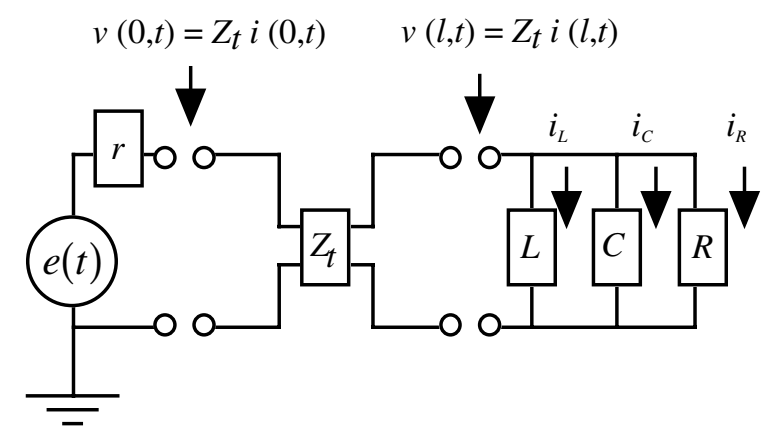

FIG. 2. Equivalent circuit of the induction unit.
TABLE I. Measured parameters for the induction units.

\begin{tabular}{ll}
\hline \hline$Z_{T}(\Omega)$ & 120 \\
$L(\mu \mathrm{H})$ & 110 \\
$C(\mathrm{pF})$ & 100 \\
$R_{C}(\Omega)$ & 330 \\
$R_{M}(\Omega)$ & 210 \\
\hline \hline
\end{tabular}

$Z_{t}$ is determined by the geometry of the power-transmission cable. $v(x, t)$ and $i(x, t)$ denote the voltage and the current at position $x$ of the cable. The circuit equation can be straightforwardly solved, assuming that (i) the cable is lossless, (ii) the characteristics of the circuit elements do not depend on frequency, and (iii) the step voltage $V_{\text {in }}$ is inputted into the upper end of the cable. Details are described in the Appendix. When there is no reflection wave, the induced voltage $v(x, t)$ can be expressed as

$$
v(l, t)=2 V_{\mathrm{in}} \frac{L \omega_{0}}{Z_{t} \sqrt{\xi^{2}-1}} e^{-\omega_{0} \xi t} \sinh \left[t \omega_{0} \sqrt{\xi^{2}-1}\right]
$$

where $\omega_{0}=1 / \sqrt{L C}$ is the intrinsic angular frequency and $\xi=\left(1 / Z_{t}+1 / R\right) /\left(2 \omega_{0} C\right)$ is the dumping factor. The voltage for a typical set of the circuit parameters given in Table I (which are close to that of the actual system) is shown in Fig. 3. Beyond the voltage peak, it almost linearly decreases, in which Eq. (1) can be approximated by $v(l, t)=V_{\text {in }}[1-R t /(2 L)]$ where $t \gg$ $2 \xi / \omega_{0}$ and $Z_{t}=R$ are supposed. The size of the droop is apparently a function of the circuit parameters. A low resistance and a large inductance are apparently helpful to reduce the size of the droop. Since the size of the cavity core, to which the inductance is proportional, is limited according to a practical reason and there is a finite resistance, a certain magnitude of the droop always remains in this type of induction device. It is noted that the droop rate is constant during acceleration, because the pulse modulator is triggered by a command signal synchronized with the revolution of a bunch to always generate the same voltage pulse.

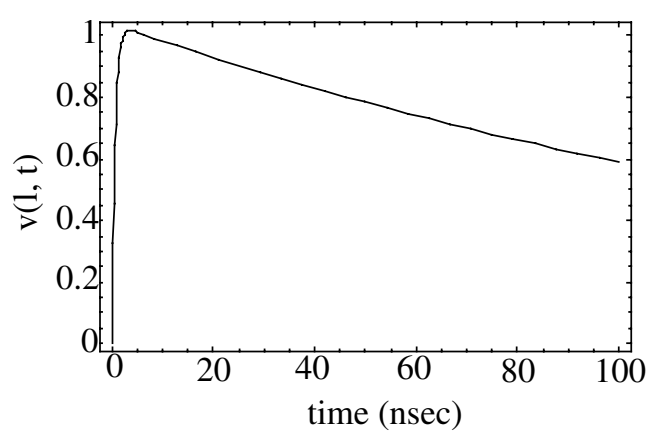

FIG. 3. Induced voltage (calculation), where $V_{\text {in }}=1(\mathrm{~V})$. 


\section{THEORY OF THE SYNCHROTRON MOTION PERTURBED BY A DROOP VOLTAGE}

According to Eq. (1), the droop in the induction voltage is intrinsic when the step voltage is inputted and has an almost negatively linear gradient. As mentioned in Sec. I, the droop voltage could cause an extra focusing and defocusing in the longitudinal motion of particles. In this section, the theory of synchrotron oscillation perturbed by a droop is developed, based on difference equations describing the longitudinal motion, which also serve for particle tracking. For the convenience of mathematical formulation, the induction units for acceleration are assumed to be placed near to the other units for particle confinement, which are rf cavities or induction units. This means that particles see two types of voltages excited in both devices at the same time in a ring.

\section{A. Difference equation for induction acceleration}

For simplicity, the barrier voltage generated in the induction gaps, which is employed for the longitudinal confinement of the superbunch, is assumed to be a step function in time,

$$
V_{c}(\phi)= \begin{cases}-V_{\text {step }} & \left(\phi<-\phi_{0}\right), \\ 0 & \left(|\phi| \leq \phi_{0}\right), \\ V_{\text {step }} & \left(\phi>\phi_{0}\right),\end{cases}
$$

where $\phi=\left\{h \int \omega_{s} d t\right\} \bmod (2 \pi)$ represents the fractional phase from a synchronous phase $(\phi=0), T_{s}=2 \pi / \omega_{s}$ is the revolution period of the synchronous particle, $h$ is the harmonic number, and $V_{\text {step }}$ is the peak voltage. The sign of the voltage must be changed beyond the transition to maintain the phase stability, just as in a conventional $\mathrm{rf}$ synchrotron. If the droop of the accelerating voltage $V_{a}$ is assumed to be linear in time as $d V_{a} / d t=k$, the accelerating voltage is written as $V_{a}(\phi)=V_{0}+k t=$ $V_{0}+h k \int_{0}^{\phi} d \phi^{\prime} / \omega_{s}$. For a synchronous particle, the temporal evolution of its energy from the $n$th turn to the $(n+1)$ th turn is given by the following difference equation:

$$
\left(E_{s}\right)_{n+1}=\left(E_{s}\right)_{n}+e V_{0},
$$

where $E_{s}$ is the energy of a synchronous particle placed at the middle point of the barrier voltage pulses. For an arbitrary particle, the corresponding equation is described by

$$
E_{n+1}=E_{n}+e\left[V_{c}\left(\phi_{n}\right)+V_{a}\left(\phi_{n}\right)\right] .
$$

Thus, the energy difference from $E_{s}, \Delta E=E-E_{s}$, satisfies

$$
\Delta E_{n+1}=\Delta E_{n}+e\left[V_{c}\left(\phi_{n}\right)+h k \int_{0}^{\phi_{n}} \frac{d \phi^{\prime}}{\left(\omega_{s}\right)_{n}}\right] .
$$

Meanwhile, the temporal evolution of the fractional phase for the particle of interest is well known to be given by

$$
\phi_{n+1}=\phi_{n}+2 \pi \eta_{n+1} h \frac{\Delta E_{n+1}}{\beta_{n+1}^{2}\left(E_{s}\right)_{n+1}},
$$

where $\eta$ and $\beta$ are the slippage factor and the relativistic beta of the design particle, respectively. In order to manifest the pure effects of the longitudinal beam dynamics on the droop, nonlinear kinematic terms $[6,7]$ are not taken account of in this paper. A set of difference equations, Eqs. (3) and (4), describes the synchrotron oscillation with the droop and becomes a theoretical basis of the following discussion.

\section{B. Differential equation and Hamiltonian}

If the parameters, such as $\eta, \beta$, and $\omega_{s}$, are assumed to be constant in $n$, Eqs. (3) and (4) can be approximated by differential equations with respect to time as

$$
\begin{gathered}
\frac{d W}{d t}=\frac{e}{2 \pi h}\left[V_{c}(\phi)+\frac{h k}{\omega_{s}} \phi\right], \\
\frac{d \phi}{d t}=\frac{\eta \omega_{s}^{2} h^{2}}{\beta^{2} E_{s}} W,
\end{gathered}
$$

where $W=\Delta E /\left(\omega_{s} h\right)$. From these equations, the Hamiltonian is easily evaluated and written in the following form:

$$
\begin{gathered}
H(\phi, W)=\frac{W^{2}}{2}+U(\phi), \\
U(\phi)=-\frac{e \beta^{2} E_{s}}{2 \pi \eta \omega_{s}^{2} h^{3}}\left[\int V_{c}(\phi) d \phi+\frac{h k}{2 \omega_{s}} \phi^{2}\right],
\end{gathered}
$$

where $U(\phi)$ represents the potential including effects caused by the droop. The effect originating from the droop is understood to be a simple quadratic potential there. As mentioned in Sec. I, this term changes its role beyond the transition energy. It takes defocusing before the transition and focusing after the transition from a macroscopic view. In Figs. 4 and 5, the potential and contours of the Hamiltonian are shown for both cases. The bathtublike potential for the step-barrier confinement shows double wells around $\phi= \pm \phi_{0}$ before the transition energy and a shallow hollow at its center after the transition energy, as shown in Fig. 4(a). Particles tend to be trapped in the double wells, and a global modification of the superbunch is predicted. Meanwhile, the potential for rf confinement looks like that of the moving rf bucket, because the position of the potential bottom changes with the phase. The contours of the Hamiltonian indicate that the stable points of the rf buckets exist at different locations from $2 m \pi$, where $m$ is an integer. This suggests that the above synchronous phase compensates an extra energy gain and loss due to the droop. 

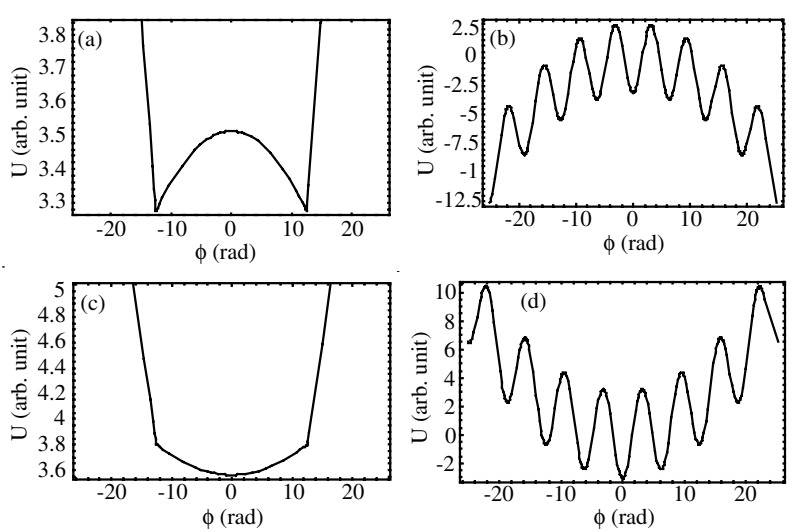

FIG. 4. Sketch of the potential for (a) the superbunch beam; (b) the rf-bunch beam before the transition energy; (c) the superbunch beam; (d) the rf-bunch beam after the transition energy. $\phi_{0}=4 \pi(\mathrm{rad}) ; k=-0.43(\mathrm{kV} / \mu \mathrm{s})$.

\section{PARTICLE TRACKING}

In order to delineate the longitudinal motion under acceleration by a long step voltage with a droop, particle tracking based on Eqs. (3) and (4) has been performed. Most of the machine parameters were taken from that of the KEK-PS, which are listed in Table II. The temporal evolution of $V_{0}$ and the kinetic energy are shown in Fig. 6, which naturally follow the field pattern of the bending magnets. Two cases of (1) step-barrier confinement and (2) conventional rf confinement were carefully examined. For the former, a rectangular pulse voltage of $85 \mathrm{kV}$ was assumed, and a confinement rf voltage of $92 \mathrm{kV}$ was assumed in the latter. For simplicity, a matching distribution to the longitudinal bucket in an injection period was assumed as an initial distribution for each confinement.
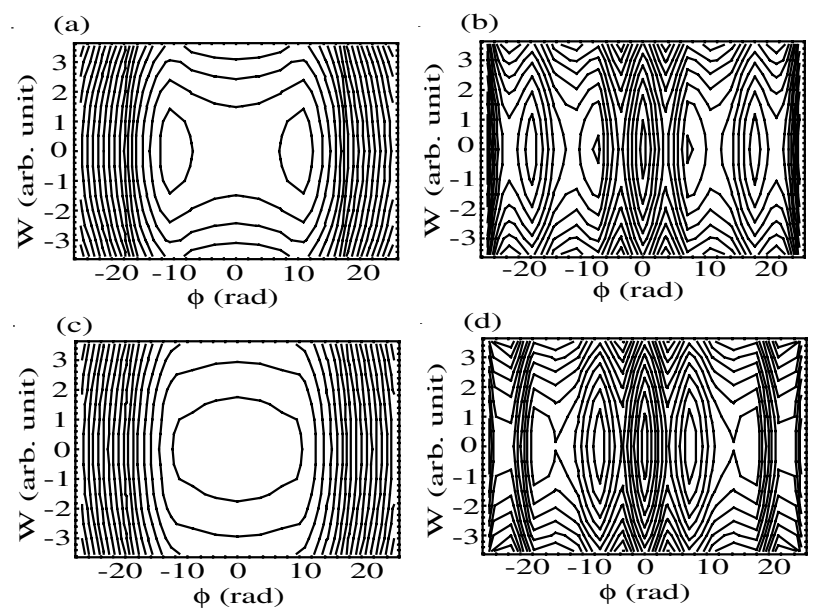

FIG. 5. Contour of the Hamiltonian for (a) the superbunch beam; (b) the rf-bunch beam before the transition energy; (c) the superbunch beam; (d) the rf-bunch beam after the transition energy. $\phi_{0}=4 \pi(\mathrm{rad}) ; k=-0.43(\mathrm{kV} / \mu \mathrm{s})$.
TABLE II. Machine parameters for the KEK-PS.

\begin{tabular}{lc}
\hline \hline Transition $\gamma$ & 6.63 \\
Circumference (m) & 339 \\
$h$ & 9 \\
Injection energy $(\mathrm{GeV})$ & 0.5 \\
Extraction energy $(\mathrm{GeV})$ & 12 \\
\hline \hline
\end{tabular}

\section{A. Transition crossing}

A novel transition crossing method, in which the particles do not see the focusing component in the confinement voltage during a short time period before and after the transition energy, was employed in the tracking. This technique has already been proposed in Refs. [1,8] and was demonstrated by computer simulations for a rf bunch [8] and a superbunch [9]. It is well known that the function of confinement and acceleration in the longitudinal direction is separated in an induction synchrotron, since the step voltages for these purposes are independently excited in different induction gaps. The induction synchrotron makes the best use of this feature at a transition crossing. In general, an intrinsic nonadiabatic feature in the synchrotron oscillation near to a transition largely deforms the rf or barrier bucket, resulting in an upright or inclined bunch shape. It has been pointed out $[1,8]$ that this bunch deformation could be substantially diminished if the focusing component in the confinement voltage is reduced to zero near a transition. The bunch never stretches in momentum space near to the transition energy. In addition, the line density can be maintained at the original level through a transition crossing. The former eliminates any concern about the momentum aperture of the accelerator ring. The latter may be helpful to suppress a fatal microwave instability caused by narrowband impedances [10,11], which distribute along the vacuum chamber. In the case of the rf synchrotron, this technique has already been demonstrated at the Fermilab Main Ring [12], where the rf focusing is eliminated during the transition crossing by the addition of the third

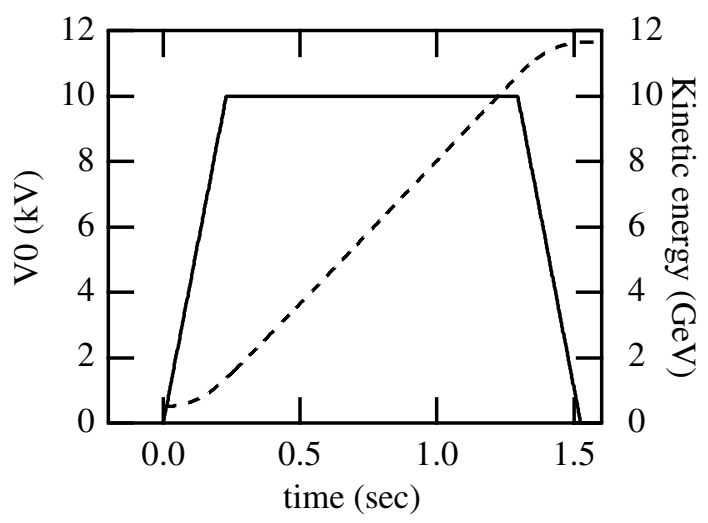

FIG. 6. $\quad V_{0}$ (solid line) and the kinetic energy (dashed line). 
harmonics rf voltage and the beam loss at the transition was completely eliminated. In the induction synchrotron, the accelerating induction voltage is required for acceleration, but it can exist without a confinement voltage if necessary.

\section{B. Superbunch}

For a superbunch, the temporal evolution of the particle distribution on phase space $(\phi, \Delta p / p)$ is shown in Figs. 7; their projections on each axis are shown in Figs. 8 and 9, in which $\Delta p / p$ represents the momentum spread. It is notable in Fig. 8(b) that the superbunch tends to split in phase space before the transition energy. As predicted by Eq. (5), the potential depth becomes deeper as $\eta$ becomes smaller. We understand that the split of the superbunch is caused by trapping a substantial fraction of particles in double wells. This is confirmed by the Poincaré map (see Fig. 10). It becomes distinct in the line density before the transition energy. However, the line density becomes flat because of a change in the potential, where all particles smoothly oscillate in the longitudinal direction. On the other hand, the complicated structure in the projection on the momentum axis is notable after the transition. This is explained by quadrupole oscillation due to the mismatching before and after the transition [see Figs. 5(a) and 5(c)]. This means that the droop effect is not time reversible about the transition. In a practical sense, there is a more significant aspect to which we must pay attention: emittance blowup. The dependence of the emittance blowup on the droop size and the length of the superbunch has been examined. The results are shown in Figs. 11. The emittance becomes larger as the droop size becomes larger, or the length becomes longer. This may be explained by the fact that the depth of the potential wells before a transition is simply proportional to the size of the droop.
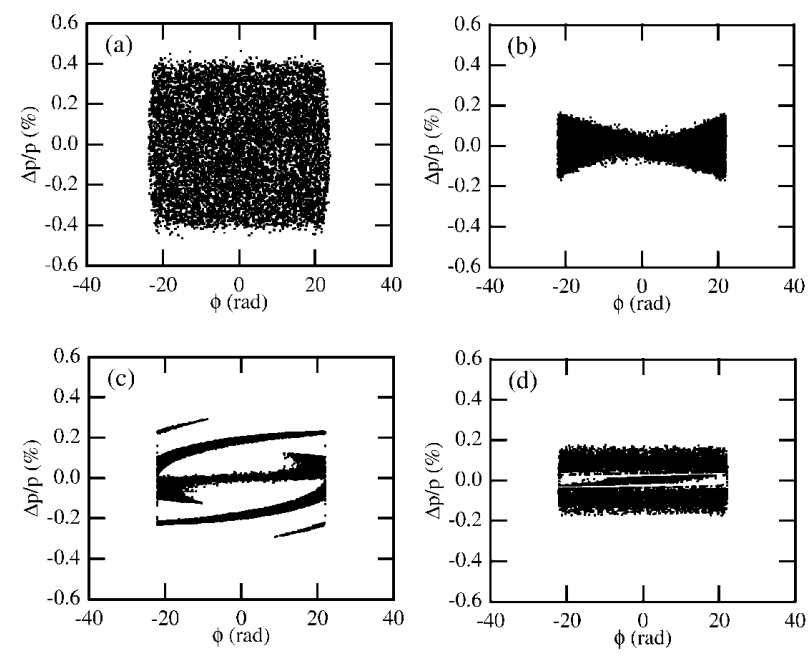

FIG. 7. Phase-space projection of a superbunch beam at (a) injection, (b) $4.69 \mathrm{GeV}$, (c) $6.69 \mathrm{GeV}$, and (d) $11.64 \mathrm{GeV} . \phi_{0}=$ $7 \pi(\mathrm{rad})$ and $k=-0.43(\mathrm{kV} / \mu \mathrm{s})$.
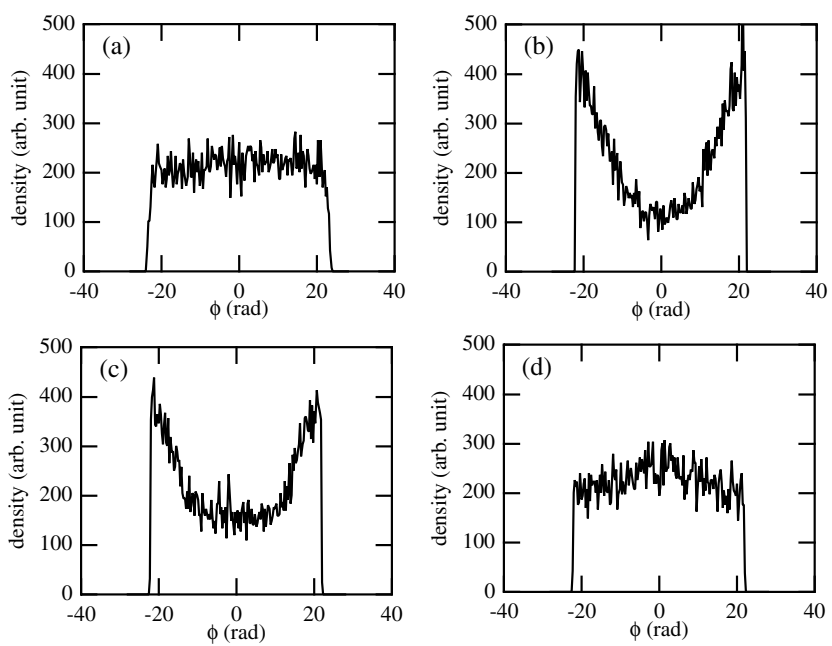

FIG. 8. Density distribution of a superbunch beam in the $\phi$ direction at (a) injection, (b) $4.69 \mathrm{GeV}$, (c) $6.69 \mathrm{GeV}$, and (d) $11.64 \mathrm{GeV}$. $\phi_{0}=7 \pi(\mathrm{rad})$ and $k=-0.43(\mathrm{kV} / \mu \mathrm{s})$.

\section{The rf bunch}

In the case of the rf bunches, the particle distribution in phase space at different acceleration energies is shown in Figs. 12, and its projection is shown in Figs. 13. No drastic phenomenon, such as in a superbunch beam, is found. This is understandable from the fact that the droop size over the bunch length is relatively small compared with the rf defocusing/focusing voltage at both sides of a transition. The emittance growths of the middle and outer bunches of seven bunches are shown in Fig. 14(a). Beyond a transition, the emittance of the outer bunch becomes slightly larger than that of the middle bunch. This comes from filamentation in the phase space induced by dipole oscillation, which is caused by a displacement of the stable point before and after the transition energy [see
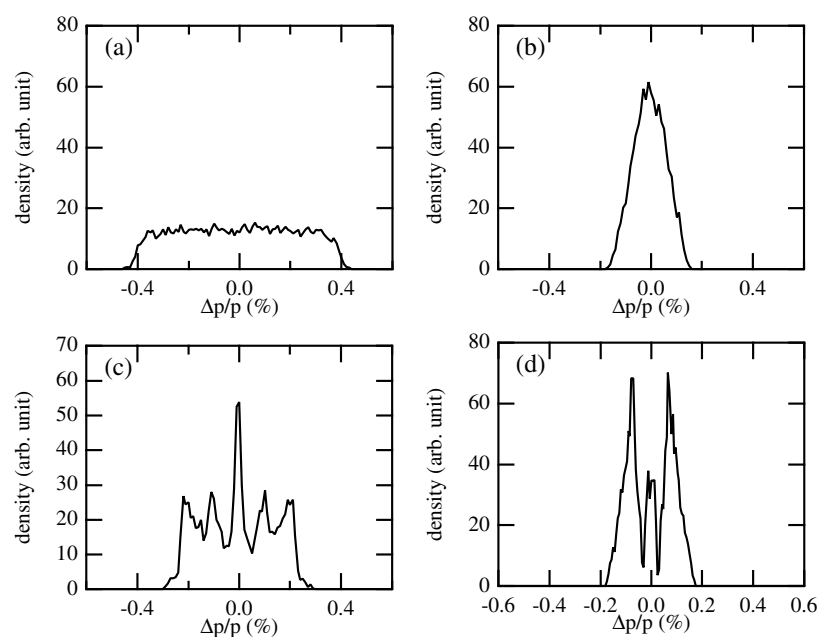

FIG. 9. Density distribution of a superbunch beam in the $\Delta p / p$ direction at (a) injection, (b) $4.69 \mathrm{GeV}$, (c) $6.69 \mathrm{GeV}$, and (d) $11.64 \mathrm{GeV} . \phi_{0}=7 \pi(\mathrm{rad})$ and $k=-0.43(\mathrm{kV} / \mu \mathrm{s})$. 


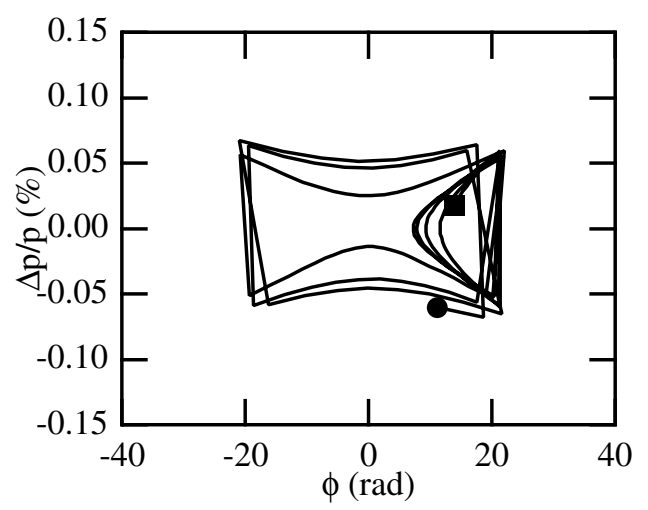

FIG. 10. Poincaré map from $1 \mathrm{GeV}$ (circle) to $3 \mathrm{GeV}$ (square).

Fig. 14(b)], as mentioned in Sec. IIIB [and see Figs. 5(b) and 5(d)]. The absolute size of the difference is still quite small. In a practical sense, this is negligible.

\section{DISCUSSION}

It has turned out that the effects of droop are serious for a superbunch and are fatal in a transition crossing. A double-well potential largely deforms the superbunch shape just after the beginning of acceleration. As the beam energy approaches the transition energy, the phase space, that is dominated by linear defocusing force, is quickly extended along the momentum axis, thus leading to a large modification of the bunch shape due to the nonadiabatic feature of the transition crossing. As suggested from the theory developed in Sec. III, the extra focusing, originating from the droop beyond a transition, changes the barrier bucket shape and becomes a substantial source of mismatching. Simulation results have indicated even splitting of the bunch shape in the momentum direction. The size of the overall emittance blowup seems to be fatal. For a practical value of the droop, these are not acceptable. In order to give the particles a fractional voltage to compensate for the droop, the introduction of an additional droop-compensating device may be indispensable for the case of a
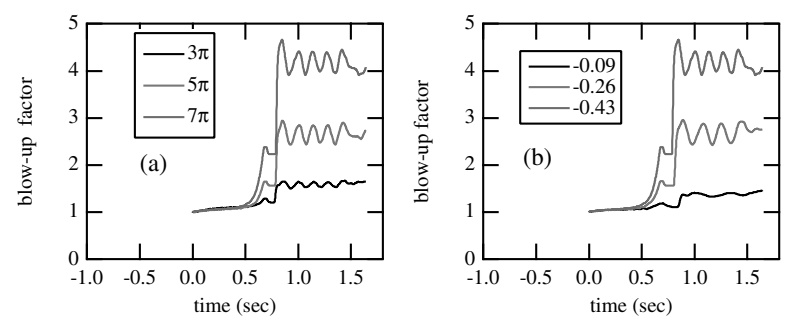

FIG. 11. Blowup factor of the emittance in the dependence on (a) $\phi_{0}$ (rad) where $k$ is set to $-0.43(\mathrm{kV} / \mu \mathrm{s})$, and (b) $k(\mathrm{kV} / \mu \mathrm{s})$ where $\phi_{0}$ is set to $7 \pi(\mathrm{rad})$. Emittance growth becomes larger as $k$ and $\phi_{0}$ become larger.
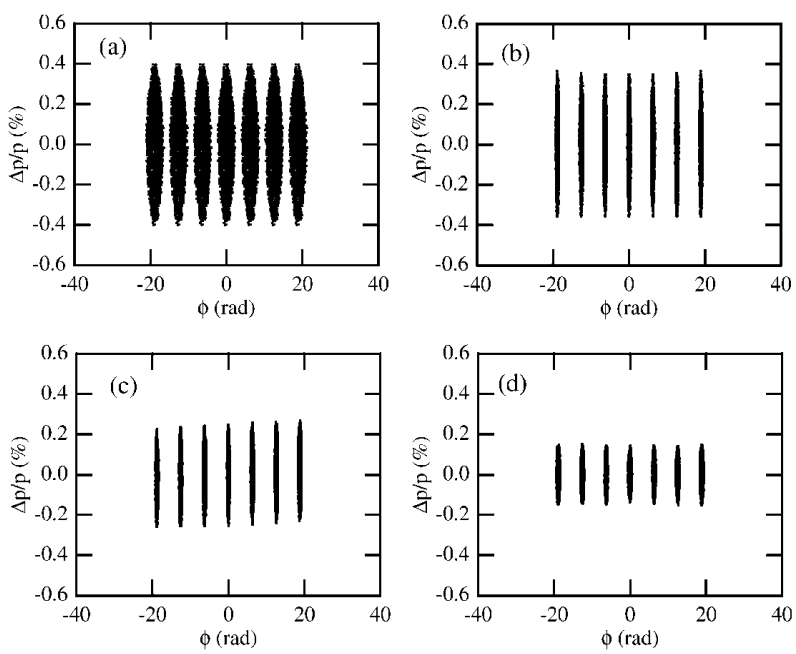

FIG. 12. Phase-space projection of the rf-bunch beam at (a) injection, (b) $4.69 \mathrm{GeV}$, (c) $6.69 \mathrm{GeV}$, and (d) $11.64 \mathrm{GeV}$. $k$ is set to $-2.00(\mathrm{kV} / \mu \mathrm{s})$.

superbunch, while the use of larger inductance material should be helpful for this purpose.

As a droop-compensating device, a technique to superimpose several pulse voltages of half sine in time [13-15] is under consideration in a realistic manner. A plural number of induction modules are stacked in an additional single induction unit. Each induction module is independently driven by its own pulse modulator. Each pulse modulator is triggered in some programmed manner with a desired voltage so that the superimposed voltage at the induction gap has a required gradient shape. An equivalent circuit for this device is shown in Fig. 15(a). This technique has been already demonstrated in a small scale, where a required linearly ramping voltage was generated, as shown in Fig. 15(b).
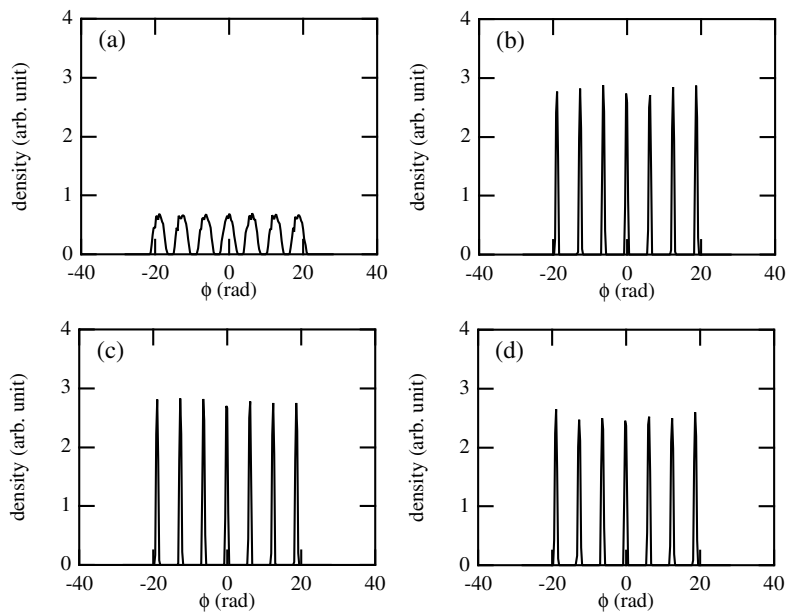

FIG. 13. Density distribution of the rf-bunch beam in the $\phi$ direction at (a) injection, (b) $4.69 \mathrm{GeV}$, (c) $6.69 \mathrm{GeV}$, and (d) $11.64 \mathrm{GeV}$. $k$ is set to $-2.00(\mathrm{kV} / \mu \mathrm{s})$. 

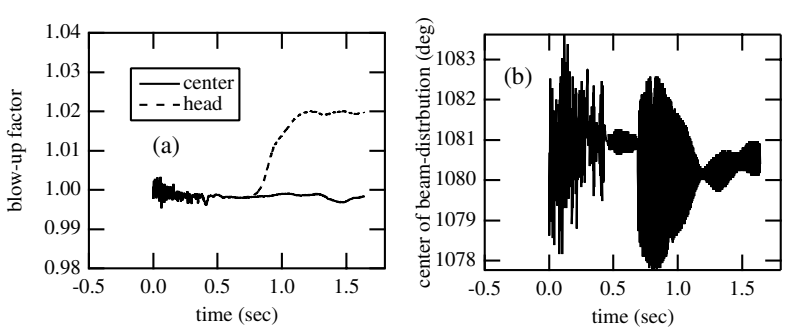

FIG. 14. (a) Blowup factor of emittance for the center and head of the rf bunch seen in Figs. 12. (b) Center of the beam distribution for the head bunch in the $\phi$ direction.

\section{CONCLUSION}

For a superbunch beam and a rf-bunch beam, the longitudinal motion during acceleration by a step voltage with droop has been theoretically analyzed and examined by multiparticle simulations, assuming the machine and beam parameters in the KEK-PS, in which the POP experiment of the induction synchrotron is scheduled. It has turned out that the droop voltage seriously affects the superbunch shape from the beginning of acceleration, and even induces particle trapping into the double-well potential originating from the droop. In the case of a synchrotron with a transition energy at the midstage of acceleration, mismatching between the largely modified bunch shape before the transition and the bucket shape after the transition leads to an extremely large emittance blowup. This fact strongly suggests the importance of a droop correction for the acceleration of a superbunch in an induction synchrotron, where particles are accelerated through the transition energy. On the other hand, its notable effects have not been observed in the case of an
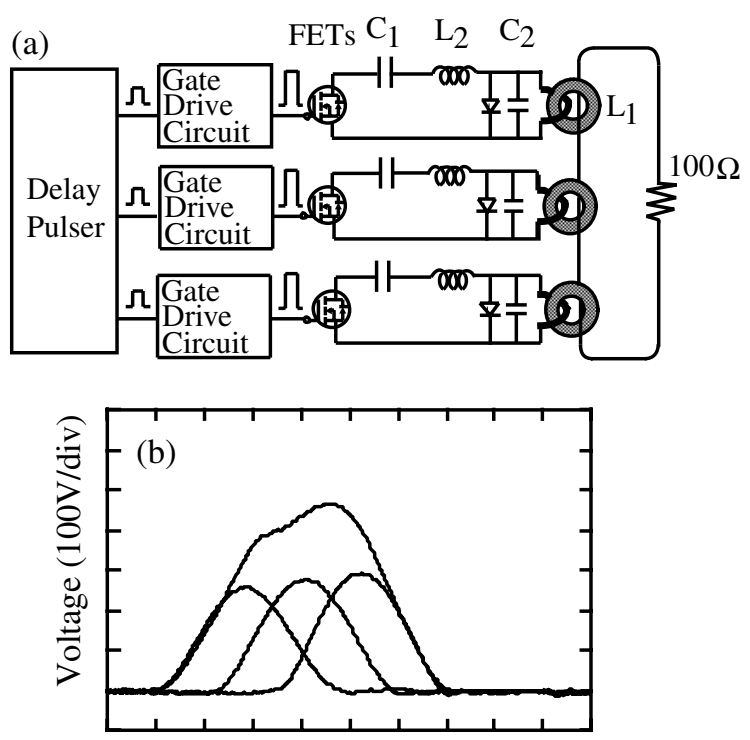

Time (200nsec/div)

FIG. 15. (a) Schematic diagram of module circuits and (b) wave form using three module circuits (measurement) [15]. rf bunch. This means that a droop correction is unnecessary for the first step of the POP experiment at the KEKPS, in which a single rf bunch will be accelerated by a $250 \mathrm{~ns}$ long induction step voltage.

\section{ACKNOWLEDGMENTS}

The authors would like to acknowledge Y. Arakida, K. Koseki, M. Wake, and others of the superbunch group for useful discussions. T. Toyama has suggested many measurements and calculations for the impedance of the cavity. One of the authors (K.T.) wishes to thank Professor Ishibashi for comments on his work. The present study has been supported by a Grant-In-Aid for Scientific Research on Priority Area (KAKENHI 140646221), a Grant-In-Aid for Exploratory Research (KAKENHI 14654048), and a Grant-In-Aid for Creative Scientific Research (KAKENHI 15GS0217).

\section{APPENDIX: DERIVATION OF EQ. (1)}

The induced voltage in an equivalent circuit of the induction unit illustrated in Fig. 2 is derived. $i_{L}, i_{C}$, and $i_{R}$ represent currents though $L, C$, and $R$, respectively. Then, the total current is written as

$$
\begin{aligned}
i(l, t) & =\frac{v^{+}(l, t)}{Z_{t}}-\frac{v^{-}(l, t)}{Z_{t}} \\
& =i_{L}(t)+i_{C}(t)+i_{R}(t),
\end{aligned}
$$

where + and - denote progressive and reflecting waves in the transmission-line theory. The induction voltage is written as

$$
\begin{aligned}
v(l, t) & =v^{+}(l, t)+v^{-}(l, t) \\
& =L \frac{d i_{L}}{d t}=\frac{\int e_{C} d t}{C}=R i_{R} .
\end{aligned}
$$

We solve these equations by the Laplace transformation in the variable $t$ to $s$. The transformed quantities are denoted by capital letters. By using Eq. (A4), Eq. (A2) is rewritten as

$$
I(l, s)=\frac{V(l, s)}{Z(s)}
$$

where $Z(s)=L R s /\left(L C R s^{2}+L s+R\right)$. Substituting Eqs. (A1) and (A3) into (A5), a well-known definition of the voltage reflection coefficient is given by

$$
\frac{V^{-}(l, s)}{V^{+}(l, s)}=\frac{Z(s)-Z_{t}}{Z(s)+Z_{t}} .
$$

Further substitution of (A6) into (A3) gives

$$
\begin{aligned}
V(l, s) & =\frac{2 Z(s)}{Z(s)+Z_{t}} V^{+}(l, s) \\
& =\frac{2 V_{\text {in }} L \omega_{0}^{2}}{Z_{t}} \frac{1}{s^{2}+2 \omega_{0} \xi s+\omega_{0}^{2}} .
\end{aligned}
$$


Here $V^{+}(l, s)=V_{\text {in }} / s$ is used, which can be evaluated from the fact that $v^{+}(l, s)$ is the step voltage. Then, Eq. (1) is given by the inverse Laplace transformation of Eq. (A7).

[1] K. Takayama and J. Kishiro, Nucl. Instrum. Methods Phys. Res., Sect. A 451, 304 (2000).

[2] K. Takayama, J. Kishiro, M. Sakuda, Y. Shimosaki, and M. Wake, Phys. Rev. Lett. 88, 144801 (2002).

[3] K. Takayama, Y. Arakida, S. Igarashi, D. Iwashita, J. Kishiro, K. Koseki, E. Nakamura, M. Sakuda, H. Sato, Y. Shimosaki, M. Shirakata, K. Torikai, T. Toyama, M. Wake, K. Horioka, and M. Shiho, in Proceedings of the PAC2003, edited by Joe Chew (IEEE, Piscataway, NJ, 2003), TPPB093.

[4] K. Torikai, Y. Arakida, S. Inagaki, J. Kishiro, K. Koseki, E. Nakamura, K. Takayama, T. Toyama, and M. Wake, in Proceedings of the PAC2003 (Ref. [3]), TPPB079.

[5] K. Koseki, Y. Arakida, S. Igarashi, S. Inagaki, J. Kishiro, E. Nakamura, H. Sato, Y. Shimosaki, M. Shirakata,
K. Takayama, K. Torikai, T. Toyama, and M. Wake, in Proceedings of the PAC2003 (Ref. [3]), ROAC011.

[6] K. Takayama, Part. Accel. 14, 201 (1984).

[7] K. Johnsen, in Proceedings of the CERN Symposium on the High Energy Accelerators and Pion Physics, edited by E. Regenstreif (CERN, Geneva, 1956), p. 106.

[8] J. Griffin and J. Maclachlan (private communication).

[9] K. Takayama, in Proceedings of Snowmass2001 (unpublished).

[10] K. Takayama, D. Arakawa, J. Kishiro, K. Koba, and M. Yoshii, Phys. Rev. Lett. 78, 871 (1997).

[11] T. Bohl, T. P. R. Linnecar, and E. Shaposhnikova, Phys. Rev. Lett. 78, 3109 (1997).

[12] C. M. Bhat, J. Griffin, J. Maclachlan, M. Martens, K. Meisner, and K. Y. Ng, Phys. Rev. E 55, 1028 (1997).

[13] M. Watanabe, M. Nakajima, and K. Horioka, Nucl. Instrum. Methods Phys. Res., Sect. A 464, 440 (2001).

[14] K. Horioka, M. Nakajima, M. Watanabe, M. Honda, E. Hotta, M. Shiho, M. Ogawa, J. Hasegawa, J. Kishiro, and K. Takayama, Laser Part. Beams 20, 609 (2002).

[15] M. Watanabe, Ph.D. thesis, Tokyo Institute of Technology (TIT), Japan, 2001 (written in Japanese). 